\title{
NUEVAS TENDENCIAS EN ESTRUCTURAS DE HORMIGON
}

\author{
(NEW TRENDS IN BUILDING CONCRETE STRUCTURES)
}

\author{
Jesús Rodriguez Santiago \\ Dr. Ingeniero de Caminos \\ Profesor Titular de Universidad

\section{GEOCISA} \\ (Los Llanos de Jeréz. 10) \\ MADRID - ESPAÑA
}

\section{RESUMEN}

Este articulo corresponde a la conferencia que con el mismo titulo impartió su autor en Madrid, el dia 2-10-90, dentro del seminario Innovaciones en la Construcción, organizada por el Institute for International Research España, S. A.

En él se resumen algunas innovaciones en el campo de las estructuras de hormigón en edificación, relacionadas con los materiales empleados (adiciones, fibras, hormigones de alta resistencia,...), con las tipologias estructurales (losas macizas armadas o postensadas, estructuras de hormigón con encofrado de poliestireno expandido,...) y con las recomendaciones para la mejora de la durabilidad de las estructuras.
SUMMARY

This paper corresponds to the lecture gave by ther author in Madrid (2-10-90), within the seminar "Innovations in Construction", organized by Institute for International Research España, S. A.

It includes a summary of some innovations in the field of building concrete structures, related to the materials (aditions, fibers, high-strength concrete,...) the type of structures (reinforced or prestressed flat slabs, concrete structures with expanded polystyrene formwork,...) and the recommendations to increase concrete structures durability.

\section{INTRODUCCIÓN}

La tecnología empleada en los diferentes sectores industriales sufre una permanente evolución, en un intento de mejorar la calidad de productos y servicios y reducir los costes de los mismos. El sector de la construcción no es ajeno a estos hechos y prueba de ello es el contenido de estas jornadas ${ }^{*}$ dedicadas a las innovaciones en la construcción.

Toda construcción requiere contar con un esqueleto o estructura resistente, integrado en la misma, que le confiera una estabilidad y rigidez adecuadas frente a las distintas acciones a las que se verá sometida a lo largo de su vida (cargas gravitatorias, acción del viento, acción sísmica,...).

* Conferencia en Madrid (2-10-90), dentro del ciclo "Innovaciones en la construcción".
A lo largo de la historia de la civilización, la tipología de las construcciones ha ido variando, empleándose distintos tipos de materiales (madera, piedra, adobe, ladrillo, acero, hormigón, plásticos,...) y distintas soluciones estructurales (muros de carga, arcos y bóvedas, elementos adintelados, pilares, vigas, losas,...).

En el momento presente, el hormigón es el material empleado con más frecuencia en las estructuras, debido a que aporta un balance adecuado entre coste y prestaciones.

En esta conferencia* se resumen algunas innovaciones en las estructuras de hormigón de los edificios, relacionadas con los materiales utilizados, con las soluciones estructurales adoptadas y con las recomendaciones para la mejora de su durabilidad. 


\section{MATERIALES}

\subsection{Componentes del hormigón}

En la composición de los hormigones se observa una clara tendencia al empleo de las adiciones y de los aditivos.

Entre las primeras, las cenizas volantes son las adiciones más utilizadas (1), aunque también se utilizan las escorias de alto horno y, últimamente, se comienza a utilizar el humo de sílice para aplicaciones específicas. Las cenizas volantes son productos sólidos en estado de fina división que proceden de la combustión del carbón pulverizado en los hogares de las centrales termoeléctricas. Prevalecen en ellas los componentes ácidos, silice y alúmina (caso de las cenizas silicoaluminosas) aunque en algunos casos la cal puede presentarse en cantidades importantes (caso de las cenizas sulfocálcicas). Tienen la capacidad de reaccionar con el $\mathrm{Ca}(\mathrm{OH})_{2}$ del cemento Portland, en presencia del agua y a temperatura ambiente, proporcionando compuestos conglomerantes que colaboran en el desarrollo de las resistencias del hormigón.

La incorporación de las cenizas al hormigón reduce su coste y mejora alguna de sus propiedades como su trabajabilidad, su impermeabilidad y su resistencia al ataque químico (sulfatos, reacción árido/álcali...), siempre que se tomen medidas adecuadas para conseguir un correcto curado del hormigón (fig. 1). Las cenizas volantes afectan a las resistencias mecánicas en el sentido de disminuirlas a edades tempranas del hormigón y aumentarlas a largo plazo.

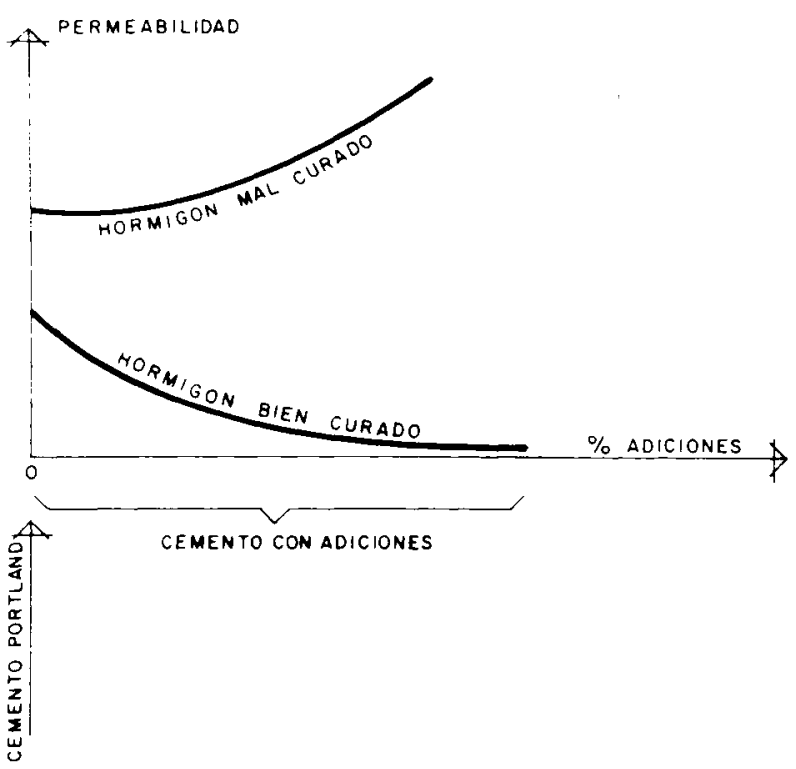

Fig. 1-Influencia del curado del hormigón, fabricado con o sin adiciones, en su permeabilidad.

(C) Consejo Superior de Investigaciones Científicas

Licencia Creative Commons 3.0 España (by-nc)
Al igual que otras adiciones, las cenizas volantes pueden emplearse como un componente más del hormigón, o mezclarse con el cemento en fábrica. A favor de la primera opción estaría el precio, y el poder elegir el cemento y la proporción cemento-cenizas más idónea para cada obra. A favor de la segunda, un posible mejor proceso de homogeneización cemento-cenizas; realizado en la fábrica de cemento. Este tema es objeto de fuertes controversias y en él inciden aspectos téc. nicos e intereses comerciales. En cualquier caso, es necesario comprobar que las cenizas tienen la calidad requerida para su empleo en la fabricación de hormigones y que se realiza un control de recepción continuo (2).

Los aditivos son productos industriales que incorporados al hormigón, antes o durante el amasado, en una proporción no superior al $5 \%$ del peso del cemento, producen la modificación de algunas de las características del hormigón, en estado fresco o endurecido. Los aditivos pueden modificar el comportamiento reológico del hormigón, actuar sobre su fraguado y endurecimiento, mejorar su durabilidad, etc. Existen aditivos específicos para el bombeo del hormigón, para morteros y hormigones proyectados, para inyecciones, etc. y puede decirse que cada vez se concibe menos un hormigón sin el empleo de los mismos.

El fabricante del aditivo debe garantizar que aquél, agregado al hormigón en las proporciones y condiciones previstas, produce la función deseada sin perturbar a las restantes caracteristicas del hormigón ni presentar peligro para las armaduras. No obstante, antes del empleo de un aditivo en la fabricación de un determinado tipo de hormigón, deben realizarse ensayos que permitan comprobar las propiedades de ese hormigón con la cantidad de aditivo dosificada (2).

\subsection{Armaduras}

El hormigón es un material con buena resistencia a la compresión pero con poca resistencia a la tracción y con comportamiento frágil. Para mejorar estas últimas propiedades, el hormigón se refuerza con armaduras de acero.

A este respecto, se observa cierta tendencia a la fabricación y al empleo de aceros soldables en estructuras de hormigón armado (3). Para cumplir la condición de soldabilidad se exigen unos contenidos máximos de carbono equivalente, carbono, fósforo y azufre del $0,54,0,24,0,055$ y $0,055 \%$, respectivamente. Por otra parte, la adherencia de estas armaduras al hormigón se controla exigiendo que cumplan un determinado valor del "indice $f_{R}$ de las corrugas", que corresponde a una determinada combinación de ciertas variables geométricas de las barras (altura y separación de los resaltos, ángulo que forman con el eje de la barra,...).

http://informesdelaconstruccion.revistas.csic.es 


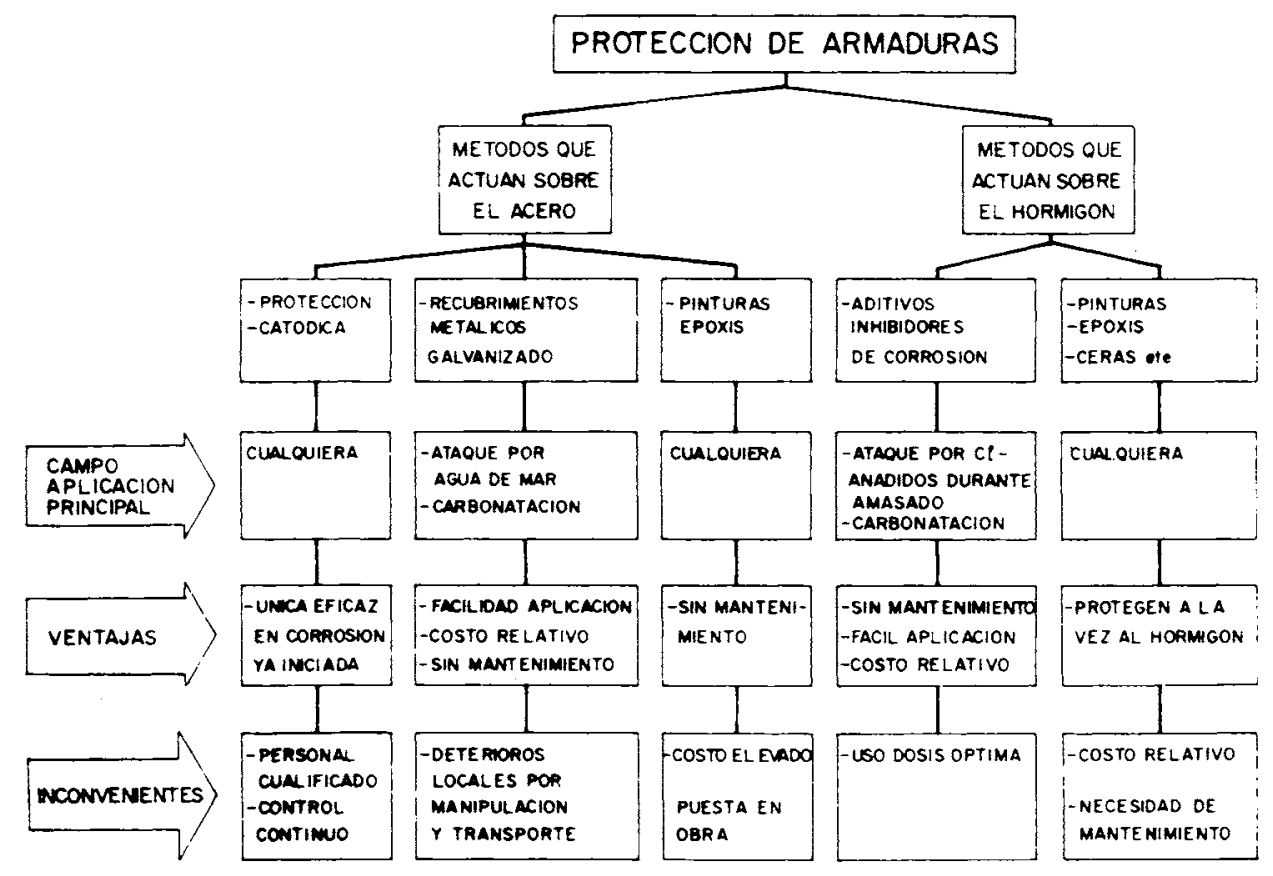

Fig. 2.- Medidas suplementarias de protección de las armaduras (5).

La fabricación de estos aceros soldables ha conducido a barras con menor ductilidad (menor alargamiento en rotura y menor relación carga de rotura/limite elás. tico). En este sentido, la tendencia europea (4) se orienta a clasificar los aceros atendiendo a sus caracteristicas de mayor o menor ductilidad y a exigir el empleo de acero de alta ductilidad para las estructuras en zonas sismicas o para aquéllas proyectadas mediante métodos no lineales de cálculo o métodos lineales con fuertes redistribucciones de solicitaciones.

Por otra parte, existe una preocupación dirigida hacia la mejora de la durabilidad de las estructuras de hormigón. En este sentido, el empleo de armaduras galvanizadas o recubiertas con resina epoxi (5), proporciona una protección adicional en determinados ambientes con riesgo de corrosión (fig. 2).

La galvanización consiste en sumergir el acero en un baño de zinc fundido a $450^{\circ} \mathrm{C}$. El acero base reaciona con el zinc y se recubre de una capa de aleaciones hierro/zinc, siendo la capa exterior de zinc puro. El zinc es mucho más estable que el acero frente a la atmósfera y también es más resistente a los cloruros. En general, el galvanizado resulta más estable con cementos bajos en álcalis y en presencia de cloruros es necesa. rio obtener un espesor de la capa de zinc puro entre 8 y $10 \mu \mathrm{m}$. La galvanización resulta ser un adecuado medio de protección cuando la corrosión es debida a la carbonatación del hormigón (reacción del $\mathrm{Ca}(\mathrm{OH})_{2}$ con el $\mathrm{CO}_{2}$ de la atmósfera), y es recomendable su uso en los prefabricados en los que el espesor del recubrimiento de hormigón suele no ser suficiente para garantizar su protección durante la vida prevista para aquéllos. La galvanización no representa un coste adicional significativo en nuestro país, proporciona una protección durante el transporte y almacenamiento de la ferralla y los desperfectos que se generen durante los mismos son fácilmente subsanables con pintura rica en zinc.

La protección de las armaduras con resina epoxi suele llevarse a cabo mediante la deposición electrostática de resina en polvo sobre las barras calentadas a una determinada temperatura. Este método de protección es una buena solución para hacer frente al ataque de la armadura por los cloruros (ambiente marino, sales de deshielo,...). Apenas se utilizan en nuestro país ya que su precio es elevado al tener que importarse. Por otra parte, algunos aspectos como la homogeneidad y durabilidad de la capa de resina epoxi, su comportamiento durante la manipulación de la ferralla y su comportamiento frente al fuego requieren mayor clarificación. No obstante, su empleo está muy extendido en USA, donde la última versión del código de cálculo de estructuras de hormigón contiene criterios especificos para el cálculo de la adherencia entre el hormigón y las armaduras con resina epoxi, exigiendo al respecto mayores longitudes de anclaje que las exigidas para las armaduras ordinarias.

Cuando se desea utilizar aceros con límite elástico superior a $600 \mathrm{Mpa}$ o cuando se quiere evitar la fisuración por tracción en el hormigón, se utiliza el hormigón pretensado. A este respecto, se pone de manifiesto el desarrollo de tendones no adherentes, aplicados a losas postensadas en forjados de edificios (ver apartado 3.2) y el desarrollo de barras fabricadas con fibra http://informesdelaconstruccion.revistas.csic.es 


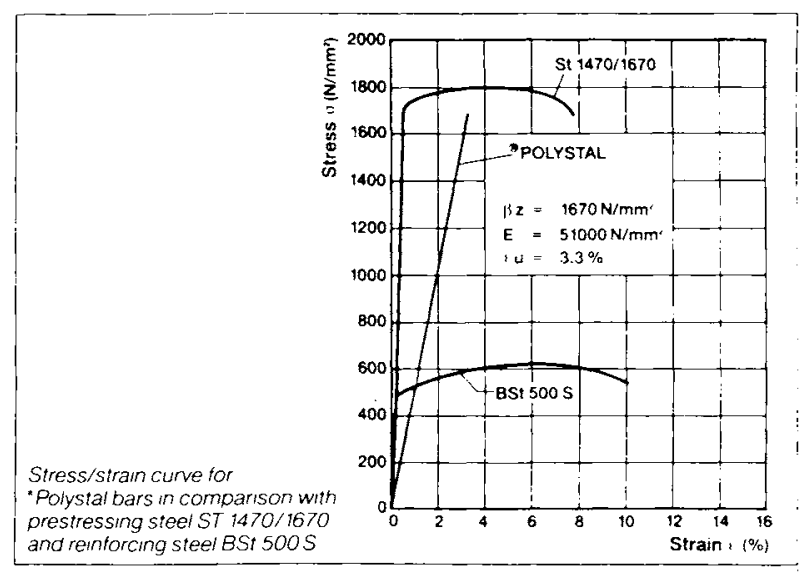

Fig. 3. - Curva tension/deformación de barras "Polystal" de fibra de vidrio utilizadas en el hormigón postensado.

de vidrio. Como ejemplo de estas últimas, se citan las barras Polystal fabricadas por BAYER que tienen 7,5 $\mathrm{mm}$ de diámetro y están formadas por 60.000 fibras de vidrio orientadas en una dirección. Los tendones están formados por 19 de esas barras y se anclan al hor. migón mediante anclajes desarrollados para este tipo de tendones por Strabag-Bau AG. Este tipo de material tiene un módulo de elasticidad de $51.000 \mathrm{Mpa}(25 \%$ del correspondiente al acero), su curva tensión-deformación hasta la rotura es una recta (fig. 3), una densidad baja $\left(2,0 \mathrm{~g} / \mathrm{cm}^{3}\right)$ y existe la posibilidad de poder integrar en las barras sensores de fibra óptica para crear tendones "inteligentes". Además, este material presenta un buen comportamiento a la fatiga y una buena resistencia a los medios agresivos.

\subsection{Fibras}

Además de reforzar los hormigones mediante barras, alambres o cables de acero que se concentran en ciertas zonas de los elementos estructurales, existe la posibilidad de reforzar aquéllos mediante fibras dispersadas en toda la masa del hormigón. Desde muy antiguo, se han mezclado fibras con determinados materiales para mejorar sus propiedades (ej: el adobe empleado en muchos pueblos de Castilla y León, obtenido mediante la mezcla de paja y barro). En el caso de los hormigones y morteros, se han empleado fibras de vidrio y metálicas, preferentemente.

Las fibras de vidrio se utilizan en la fabricación de prefabricados de GRC (morteros de cemento reforzados con fibra de vidrio) (6), habiendo sido necesario desarrollar fibras de vidrio resistentes a los álcalis del cemento. En general, el GRC no se emplea con funciones claramente estructurales pues no se ha resuelto todavía que algunas de sus características mecánicas iniciales, en especial su gran ductilidad, se mantengan constantes en el tiempo. Actualmente, se trabaja en la mejora de las fibras (composición del vidrio, acaba- dos,...) y en la modificación de la matriz de cemento mediante el empleo de aditivos (polímeros,...) y adiciones (metacaolín, cenizas volantes,...).

Las fibras de acero se han empleado en la fabricación de morteros y hormigones, con el fin de mejorar su tenacidad, resistencia al impacto y resistencia a lafisuración, principalmente (7). Se han utilizado en firmes de hormigón de aeropuertos y carreteras, en gunitados de túneles y en hormigones de fachadas, entre otras aplicaciones. En general, no es económico sustituir la armadura convencional por las fibras, empleándose éstas como complemento de aquélla (ej.: mejora de la ductilidad de los nudos en pórticos de hormigón armado para construcciones en zonas de alto riesgo sismico). Sin embargo, se emplea como sustitutivo de la armadura convencional para refuerzos frente a la fisuración o cuando la colocación de la armadura convencional es muy costosa, a causa del procedimiento constructivo empleado (ej:: mejora de la capacidad a cortante de viguetas prefabricadas con "ponedoras", en las que no suele ser posible la disposición de armadura transversal).

Habitualmente, se emplean dosificaciones entre 30 y $80 \mathrm{~kg} / \mathrm{m}^{3}$ con fibras rectas o con fibras con formas especiales para mejorar su adherencia al hormigón. Para aplicaciones especiales en medios agresivos se emplean fibras galvanizadas.

Recientemente, ha sido publicada normativa especifica para este tipo de hormigones (serie 83.500 de las normas UNE) que contiene tanto especificaciones de los materiales como métodos de ensayo para valorar sus propiedades (trabajabilidad mediante el cono invertido, medida de la tenacidad en flexión,...), lo que sin duda facilita el empleo de este material.

En los últimos años, han comenzado a utilizarse también fibras de polipropileno, que a pesar de tener una deformabilidad mayor que el hormigón, mejoran su comportamiento a la fisuración, al evitar la formación de microfisuras en las primeras edades del hormigón. Actualmente, el mercado ofrece un amplio abanico de tipos de fibras (carbono, metal amorfo, acrílicas,...), además de las ya citadas, que hacen posible cada dia un empleo mayor de los hormigones con fibras. Esta utilización se verá sin duda incrementada cuando la normativa vigente contemple los criterios para el cálculo de este tipo de materiales.

\subsection{Hormigones de alta resistencia}

Hoy en dia se fabrican con cierta facilidad hormigones con resistencias caracteristicas a compresión entre 40 y $60 \mathrm{Mpa}$, siempre que se cuente con unos áridos y un cemento adecuados. Además, en algunos países (USA, http://informesdelaconstruccion.revistas.csic.es 
Noruega y Suecia) se consiguen hormigones con resistencias superiores a $100 \mathrm{Mpa}(8)$.

Este tipo de hormigones tiene su campo de aplicación en construcciones de ingenieria civil (plataformas maritimas, puentes,...) y en edificación (edificios con gran número de pisos), siendo sus ventajas principales:

- Disminución de la deformabilidad al aumentar el valor del módulo de deformación longitudinal. Además, la retracción y la influencia pueden reducirse hasta un $50 \%$ con respecto a las de los hormigones convencionales.

- Mejora de la durabilidad del hormigón y sus armaduras, debido a la estructura porosa del mismo y al elevado contenido de cemento. Mejora también de su comportamiento ante la abrasión y ante la acción del hielo.

- Aumento de la velocidad de construcción de las estructuras, al conseguirse resistencias iniciales que permiten desencofrarlas a edades muy tempranas.

- En edificios en altura, reducción de los efectos del acortamiento de pilares, reducción del coste de las cimentaciones, disminución de las deformaciones ante la acción del viento y mejora del amortiguamiento frente a acciones dinámicas.

Sin embargo, estos hormigones tienen un comportamiento más frágil (fig. 4), aunque dicho comportamiento puede mejorarse mediante un "detailing" muy cuidadoso (zunchado con armaduras, zunchado exterior con chapas en pilares compuestos,...) o mediante el empleo de fibras.

La utilización de estos hormigones requiere que se desarrollen códigos que contengan criterios para su fabricación y para el cálculo de las estructuras construidas con ellos (9), ya que los códigos existentes no cubren este tipo de hormigones.

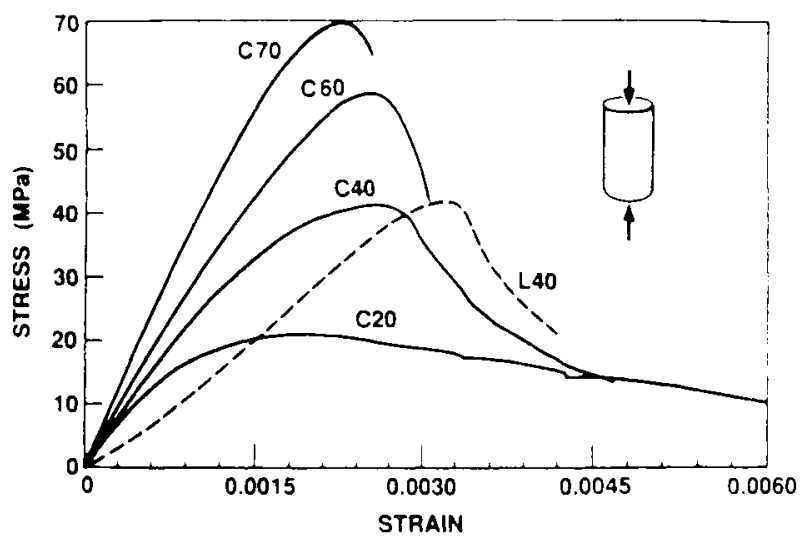

Fig. 4.-Curva tensión/deformación de hormigones con distintas re. sistencias a compresión.

(c) Consejo Superior de Investigaciones Científicas

Licencia Creative Commons 3.0 España (by-nc)
Actualmente, se continúa investigando sobre aspectos relacionados con el diseño y trabajabilidad de las mezclas, con el comportamiento del hormigón bajo cargas mantenidas o bajo cargas ciclicas (fatiga), etc.

A titulo de ejemplo, se incluyen datos referentes a uno de los hormigones empleados en la construcción del Arco de la Defensa de Paris (9), colocado mediante bombeo en zonas con fuerte densidad de armaduras (hasta $300 \mathrm{~kg} / \mathrm{m}^{3}$ ):

\section{DOSIFICACION POR $\mathrm{m}^{3}$ DE HORMIGON}

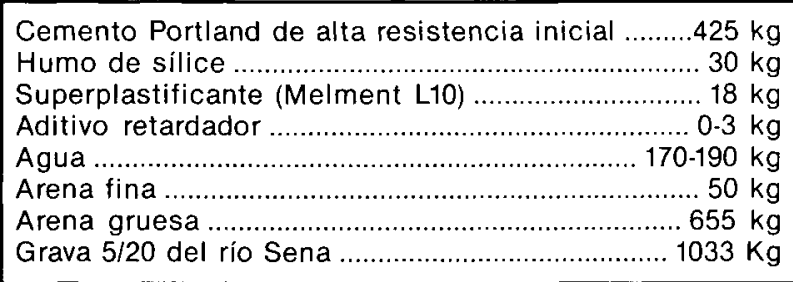

\section{CARACTERISTICAS DEL HORMIGON}

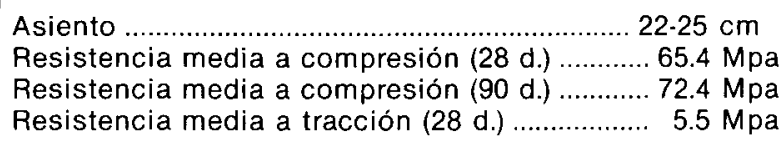

Finalmente, se citan algunas estructuras recientes en las que se han empleado hormigones de alta resistencia, (9):

\begin{tabular}{|l|lllc|}
\cline { 2 - 5 } \multicolumn{1}{l|}{ EDIFICIOS } & LUGAR & AÑO & PISOS & $\mathbf{R}_{\mathbf{2 8}}$ (Mpa) \\
\hline 900 N. Michigan Annex & Chicago & 1986 & 15 & 97 \\
South Wacker Tower & Chicago & 1989 & 79 & 83 \\
Grande Arche de la Defense Paris & 1988 & - & 65 \\
Two Union Square & Seattle & 1989 & 58 & 115 \\
Pacific First Center & Seattle & 1989 & 44 & 115 \\
Gateway Tower & Seattle & 1989 & 62 & 94 \\
\hline
\end{tabular}

\begin{tabular}{|lcccc|}
\cline { 2 - 5 } \multicolumn{1}{l|}{ PUENTES } & LUGAR & AÑo & LUZ MAX. & $\mathbf{R}_{\mathbf{2 8}}$ (Mpa) \\
\hline Pont du Pertuiset & Francia & 1988 & $110 \mathrm{~m}$ & 65 \\
Giske & Noruega & 1989 & $52 \mathrm{~m}$ & 55 \\
Boknasundet & Noruega & 1990 & $190 \mathrm{~m}$ & $60^{\star}$ \\
Helgelandsbrua & Noruega & 1990 & $425 \mathrm{~m}$ & 65 \\
\hline
\end{tabular}

(*) Hormigón ligero

\section{ESTRUCTURAS}

\subsection{Estructuras con losas macizas de hormigón armado}

A principios de los años setenta, se comenzaron a construir estructuras formadas por muros y losas macizas de hormigón armado (10), gracias a la combinación del uso de grandes encofrados con la utilización de técnicas de curado acelerado del hormigón, en un 


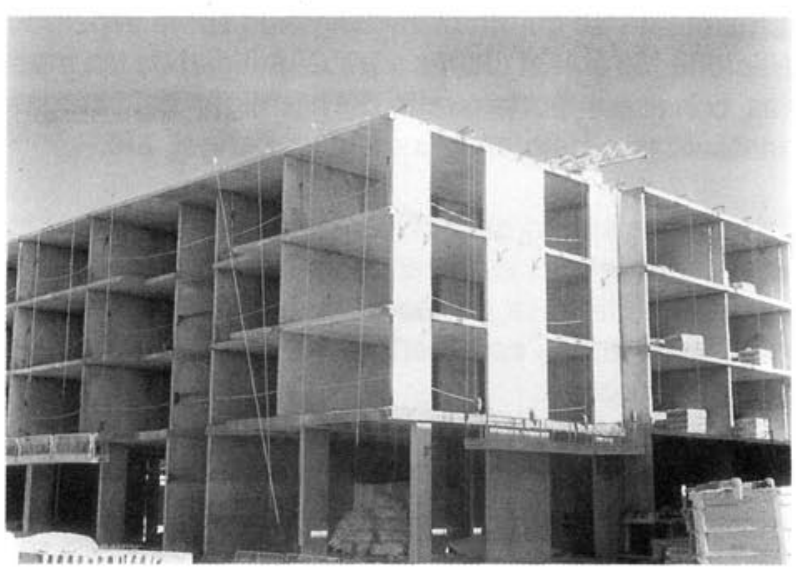

Fig. 5.-Estructura de un edificio con muros y losas de hormigón ar. mado.

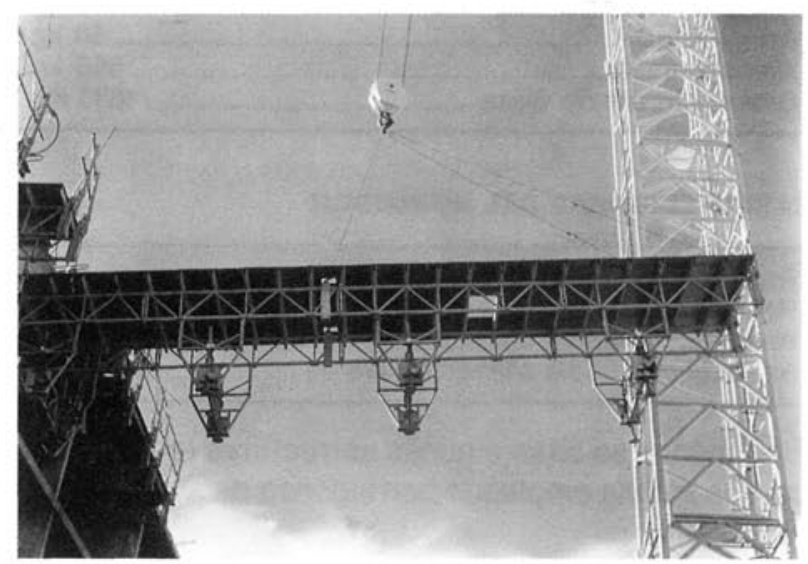

Fig. 6.-Mesa empleada como encofrado de las losas.

intento de disminuir la incidencia de la mano de obra en la construcción de edificios.

Los muros de hormigón se encofran con grandes paneles metálicos y sustituyen a una parte de la tabiqueria y los cerramientos del edificio. En ellos se alojan los huecos para las puertas y ventanas (fig. 5). Los forjados se resuelven mediante losas macizas para cuyo hormigonado se utilizan grandes encofrados "tipo mesa" de 20 a $60 \mathrm{~m}^{2}$ de superficie cada uno (fig. 6).

Los muros se sitúan en dos direcciones y de forma que, sustituyendo a la mayor cantidad posible de cerramien. tos y tabiqueria, permitan el movimiento de las mesas durante la construcción de los forjados.

Al utilizar elementos estructurales de gran superficie se intenta reducir en lo posible sus espesores con el fin de optimizar el consumo de hormigón. Los muros se proyectan con espesores de $12 \mathrm{a} 15 \mathrm{~cm}$ y se ubican a distancias entre si de 3,00 a $4,50 \mathrm{~m}$, con el fin de poder salvar estas luces mediante losas de 14 a $16 \mathrm{~cm}$ de canto. Los espesores y cantos de estos elementos deben ser suficientes para evitar problemas de inesta. bilidad en los muros o de excesiva deformabilidad en los forjados.
Algunos muros terminan en su planta inferior en pilares apantallados para asi resolver mejor los locales comerciales o zonas de porches de la planta baja. Este debilitamiento de los muros reduce en gran medida su rigidez y es preciso tenerlo en cuenta al distribuir las solicitaciones debidas a las acciones horizontales entre todos los muros del edificio.

En la construcción de los muros y las losas se requiere una buena colocación de la ferralla, ya que pequeños errores en su emplazamiento produce variaciones porcentuales importantes en el valor de los cantos útiles respecto a los considerados en los cálculos, al ser todos los elementos de espesor pequeño.

El hormigón de los muros debe tener un tamaño máximo del árido inferior a $15 \mathrm{~mm}$ y una consistencia plástica obtenida con una relación agua/cemento moderada y el uso de superplastificantes. Es necesario controlar el hormigonado de las zonas próximas a los huecos, ya que la presencia de armaduras de refuerzo de los negativos de los encofrados y de las instalaciones eléctricas embebidas en los muros lo dificultan en gran medida.

La necesidad de reutilizar los grandes encofrados obliga a plantear un desencofrado rápido de los muros y forjados empleando sistemas de curado acelerado y en muchos casos cementos de alta resistencia inicial. Los muros se desencofran antes de las 24 horas, ya que el curado del hormigón resulta acelerado por el propio calor de hidratación unido al empleo de encofrados isotermos (fig. 7). Los forjados suelen curarse mediante la aplicación de calor con mantas eléctricas con lo que se consigue desencofrar al dia siguiente de su hormigonado. Para esto, es preciso controlar la evolución de la resistencia del hormigón en las primeras veinticuatro horas, fabricando a tal efecto probetas con el hormigón empleado en el forjado, que se conservan en las mismas condiciones ambientales que las losas.

La terminación del hormigón de los muros y losas debe permitir la aplicación directa de las pinturas o revestimientos de acabado por lo que interesa cuidar las uniones entre los diferentes encofrados y emplear desencofrantes compatibles con los acabados previstos.

La solución anteriormente descrita es a veces incom. patible con la necesidad de disponer de espacios diá. fanos en los edificios. Asi, se plantea una tipología estructural alternativa en la que se mantiene la losa de hormigón y se sustituyen todos o parte de los muros por pilares de hormigón armado.

En esta solución las losas se encofran también con mesas y los pilares con encofrados formados por dos diedros metálicos de longitud igual a la altura entre las plantas (fig. 8). Suelen igualarse las escuadrias de los pilares de una misma alineación en cada planta con el http://informesdelaconstruccion.revistas.csic.es 


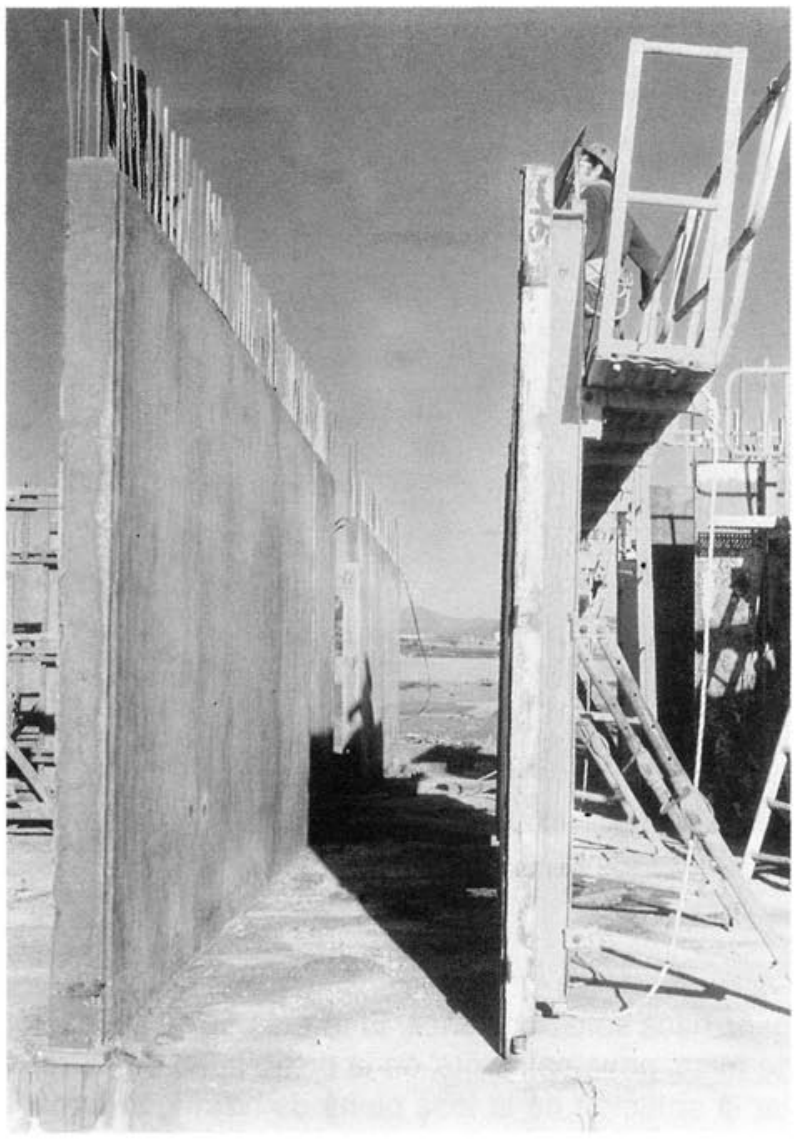

Fig. 7. - Banche empleado como encofrado de los muros.

fin de simplificar el encuentro de las mesas con ellos. Las secciones transversales de los pilares se mantienen sin variación en dos o tres alturas con el fin de reducir las operaciones de adaptación de los encofrados al cambiar de planta en el edificio.

Al igual que en el caso anterior, es preciso reducir en lo posible el canto de la losa con el fin de disminuir la cantidad de hormigón. Se utilizan, generalmente, cantos comprendidos entre 0,14 y $0,18 \mathrm{~m}$, lo que permite salvar distancias inferiores a $6,00 \mathrm{~m}$ con esbelteces comprendidas entre el veinticincoavo y el treintaavo de la luz. Sin embargo estas esbelteces pueden generar toda una problemática en las uniones de los pilares con las losas, exigiendo fuertes concentraciones de armaduras que pueden dificultar su hormigonado.

El armado de las losas a flexión se realiza con mallas y las zonas sobre pilares y los centros de vanos se refuerzan con redondos. La presencia de huecos, especialmente cerca de los pilares, requierre unos refuerzos localizados que deben definirse en proyecto.

El desencofrado de las losas a edades del hormigón muy tempranas requiere los controles ya mencionados en la anterior tipologia de estructuras. Cabe aqui añadir que las zonas de los forjados situadas sobre los pi-

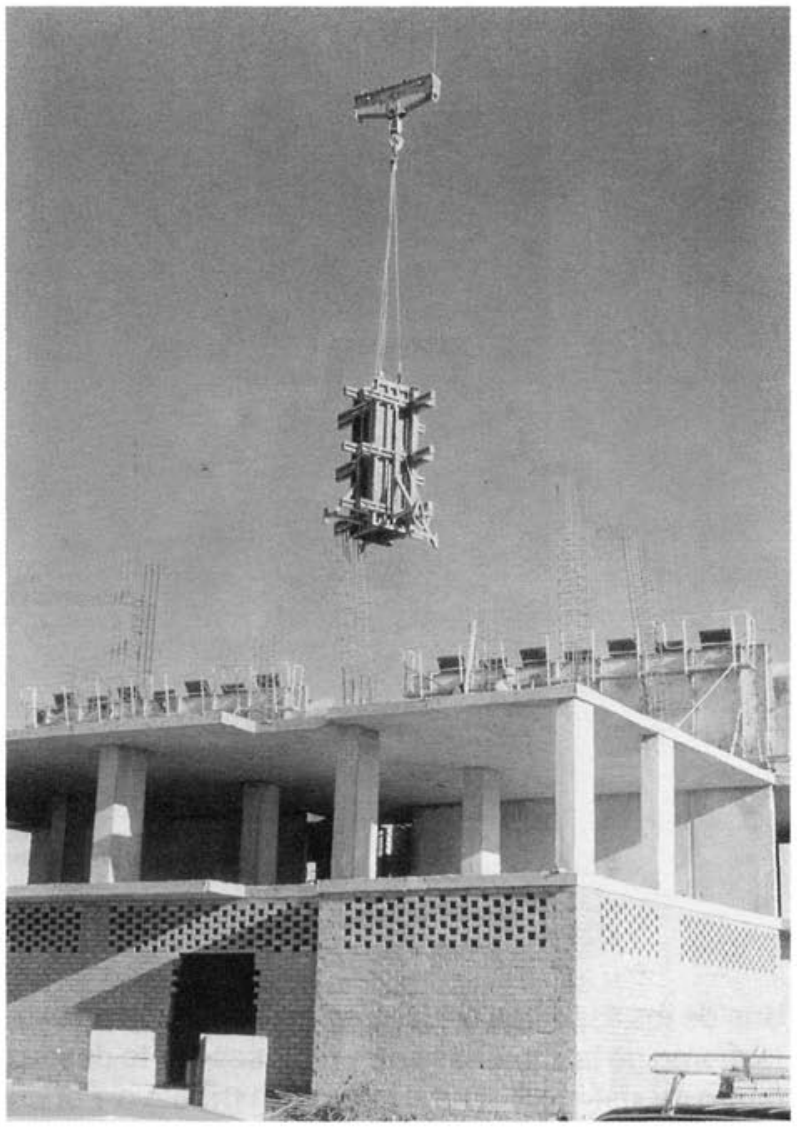

Fig. 8. - Encofrado para pilares.

lares (las más solicitadas) son las que a veces resultan peor curadas, al no ser fácil cubrirlas con las "mantas" ya que las armaduras de espera de los pilares lo dificultan. Es muy importante controlar este aspecto para evitar que las losas, al retirar las mesas, sufran importantes daños en la unión con los pilares, sea por un problema de flexión o de punzonamiento.

Los forjados de las dos soluciones anteriores estaban formados por losas macizas de canto constante que se hormigonaban in situ, utilizando como encofrados unas "mesas" de gran superficie. Puede también plantearse esta misma tipología para el forjado, fabricando las losas de manera diferente, si el uso de las mesas no es posible.

Este es el caso en el que, primeramente, se prefabrican unas losas de canto menor al total, en una instalación de prefabricación a pie de obra o en una instalación independiente de ésta. Estas losas, Ilamadas "pre. losas", se trasladan a su ubicación definitiva en el edificio cuando el hormigón ha adquirido la resistencia adecuada. Antes de colocar la armadura restante y completar el hormigonado del forjado hasta su canto total, se sitúan los puntales o sopandas necesarios para que las prelosas puedan resistir el peso del hormigón fres. co y las cargas derivadas de su puesta en obra (11).

http://informesdelaconstruccion.revistas.csic.es 


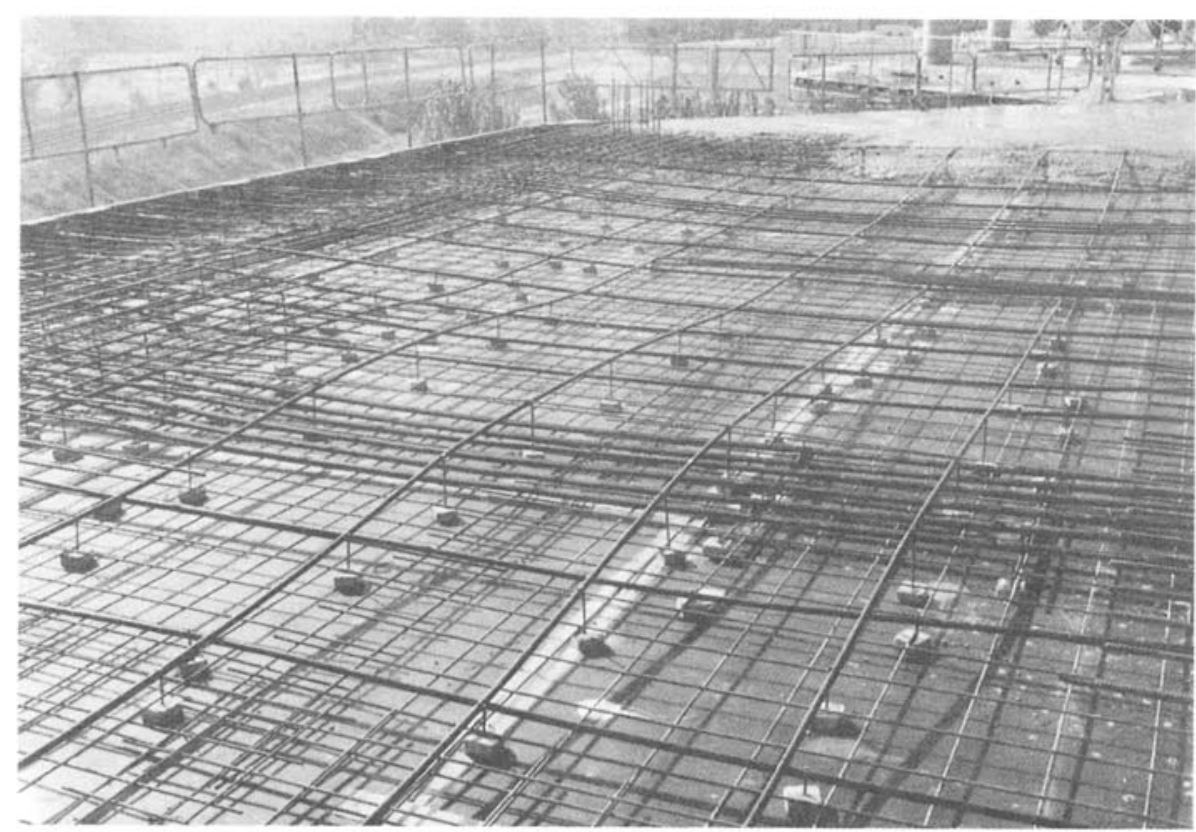

Fig. 9.-Detalle del trazado de cabies en losas postensadas.

Uno de los aspectos criticos de esta solución es el tratamiento de las uniones entre prelosas, tanto desde el punto de vista estructural como constructivo. Por otra parte, la ejecución, acopio y transporte de las prelosas debe planificarse de manera que éstas no estén sometidas a esfuerzos superiores a los previstos en el cálculo.

\subsection{Estructuras con losas macizas de hormigón postensado}

Aunque se han construido ya más de 30 millones de $\mathrm{m}^{2}$ de forjados con losas postensadas en USA (12), esta solución constructiva ha comenzado a utilizarse en Es-

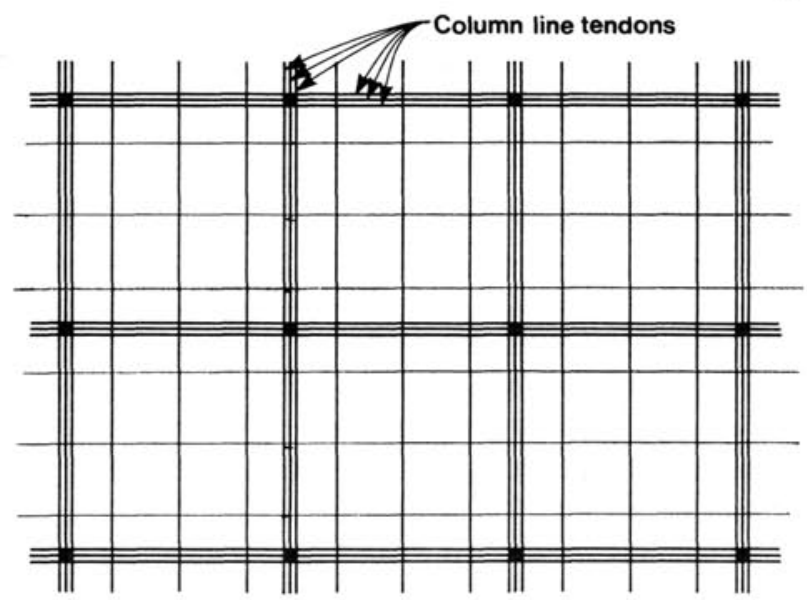

Fig. 10. - Replanteo de cables en la planta de losas postensadas (c) Consejo Superior de Investigaciones Científicas Licencia Creative Commons 3.0 España (by-nc) paña hace sólo unos años. El interés de su aplicación se basa, principalmente, en la posibilidad de extrapolar la solución de la losa plana de hormigón armado a estructuras con mayores distancias entre sus pilares. Ello es debido a que, mientras en hormigón armado se emplean esbelteces "luz/canto" de 25 a 30 , en las losas postensadas se emplean esbelteces de 45 a 50.

La armadura activa está formada por tendones no adherentes con trazado curvo en alzado (fig. 9), para adaptarse mejor a las necesidades estructurales. En planta, los cables suelen tener trazado recto y se sitúan en dos direcciones ortogonales, con distribucciones uniformes o concentrándolos sobre las alineaciones de pilares (fig. 10).

Los tendones suelen ser cables con diámetros 0,5 ó 0,6 " que se introducen en vainas de polietileno, con una grasa entre cable y vaina que facilita el deslizamiento del cable durante su tesado y proporciona protección al mismo. Los tendones se anclan en sus extremos mediante cuñas contra placas de acero de pequeñas dimensiones ( $80 \times 140 \mathrm{~mm}$, aproximadamente).

Las principales ventajas de este tipo de estructuras son:

- Las estructuras son más ligeras y requieren cimen. taciones menores.

- Se obtiene un aprovechamiento mejor del espacio interior de los edificios, al emplear cantos pequeños en los forjados y distancias grandes entre pilares.

- Ausencia de fisuración en las losas y flechas peque. ñas.

http://informesdelaconstruccion.revistas.csic.es 
Además de la armadura activa, es preciso disponer armadura pasiva como complemento a la anterior, especialmente si se emplea el pretensado parcial, siendo obligatorio disponer una cuantía mínima por razones de ductilidad y de control de la fisuración.

Existen recomendaciones para el proyecto de estas estructuras (13) (14) para las que, en general, se recomienda limitar la compresión media en el hormigón de la losa a 3 ó $4 \mathrm{Mpa}$, salvo que se realicen estudios para tener en cuenta los efectos del acortamiento de la losa en el resto de la estructura. Por otra parte, se admite el empleo del método de los pórticos virtuales como procedimiento simplificado para la obtención de los esfuerzos.

Las losas postensadas pueden salvar luces importantes, estando limitadas por la capacidad de la unión pilar!losa a punzonamiento. Ésta puede mejorarse si se disponen armaduras transversales, de difícil colocación y de dudosa colaboración en losas con cantos pequeños, o se disponen ábacos o capiteles entre el pilar y la losa.

Como ejemplo de estas estructuras, se cita unos edificios de oficinas construidos recientemente por DYC en Madrid. Son edificios de 9 plantas, formadas por tres octógonos de $600 \mathrm{~m}^{2}$, unidos entre si por uno de sus lados. Se proyectó una losa de $25 \mathrm{~cm}$ de canto, para salvar luces superiores a $9,0 \mathrm{~m}$, sometida a $1,1 \mathrm{t} / \mathrm{m}^{2}$ de carga total. Se diseñaron tres familias de cables, dos en direcciones perpendiculares y otra dispuesta en el perímetro exterior de los octógonos. Se emplearon los materiales siguientes:

- Hormigón: Tipo H-300. Cemento tipo II-45: 400 kg/m33. Relación $\mathrm{A} / \mathrm{C}=0,39$. Aridos: $0 / 5$ y 5/20.

- Cables: Diámetro 0,6". Tensión de rotura 1860 Mpa.

- Cuantias: Armadura activa: $\quad 5,7 \mathrm{~kg} / \mathrm{m}^{2}$ Armadura pasiva: redondos: $7,4 \mathrm{~kg} / \mathrm{m}^{2}$ mallazo: $4,0 \mathrm{~kg} / \mathrm{m}^{2}$

La cuantía de armaduras empleadas es algo elevada debido a la forma de las plantas octogonales, en las que ha habido que emplear muchos refuerzos en bordes exteriores y en huecos.

La construcción de la estructura se realizó a razón de un octógono $\left(600 \mathrm{~m}^{2}\right)$ cada tres días. El tesado de los cables se realizó con gatos Alevín, manejados por un solo operario, y la fuerza de tesado fue de $21 \mathrm{t}$.

\subsection{Estructuras de hormigón con encofrados de poliestireno expandido}

En los últimos años han aparecido soluciones constructivas en las que se emplea poliestireno expandido como encofrado perdido para la ejecución de los cerramientos y la estructura de los edificios. Con la utilización del poliestireno se logra un buen aislamiento térmico en los edificios y, por otra parte, se manejan paneles muy ligeros durante la construcción de los mismos. Entre estas soluciones, se cita el sistema Dragados-Plastbau (fig. 11), formado por muros portantes en dos direcciones y por forjados nervados (15) (16) (17).

Los muros portantes se construyen a partir de unas planchas de poliestireno aligerado de 0,22 $\mathrm{m}$ de espesor y de una longitud igual a la altura entre plantas. Los aligeramientos permiten ubicar en su interior pilares de hormigón en masa o armado, de 0,15×0,16 m de sección, a una distancia entre si múltiplo de $0,30 \mathrm{~m}$. Los muros se rematan con vigas zuncho a la altura de los forjados.

La preparación de los paneles de los muros portantes se inicia en factoria, cortando con un hilo incandescente las planchas macizas de poliestireno de $15 \mathrm{~kg} / \mathrm{m}^{3} \mathrm{de}$ densidad. Realizando este corte con quiebros (tipo viga metálica aligerada), se conforman dos mitades que debidamente presentadas entre sí constituyen el perfil hueco de poliestireno. La fabricación en factoría termina con la colocación de mallas de acero, a ambos lados del panel, cosidas entre sí mediante redondos soldados que lo atraviesan de lado a lado.

El hormigonado de los pilares se lleva a cabo con los paneles situados en su posición definitiva, una vez realizada la primera fase de proyección del microhormigón por ambas caras del panel, para resistir el empuje del hormigón fresco. La segunda fase de proyección del microhormigón de los muros se realiza cuando están ya colocados los forjados. Éstos se construyen a partir de unas placas de yeso-cartón sobre las que se pegan piezas de poliestireno expandido que sirven de aligeramiento y encofrado para el hormigonado de los nervios de hormigón, que se lleva a cabo en dos etapas. En la primera, se hormigonan los nervios hasta una determinada altura para consguir una cierta autoportancia. En la segunda, que se lleva a cabo con el emplazamiento definitivo del forjado, se hormigonan las vigas de los muros y el resto del forjado, conjuntamente con los pilares de los muros.

Esta tipología estructural está concebida para edificios de hasta cuatro o cinco plantas, hormigonándose, en cada caso, el número de pilares requerido según los cálculos realizados. Es necesario disponer muros en las dos direcciones a fin de hacer frente a las acciones horizontales del viento o del sismo.

El cálculo de los muros se lleva a cabo según la teoría general del hormigón. Los pórticos, formados por las vigas y pilares de los muros, resisten las cargas gravitatorias y se confía a las capas de microhormigón el arriostramiento de los edificios de poca altura no sihttp://informesdelaconstruccion.revistas.csic.es 


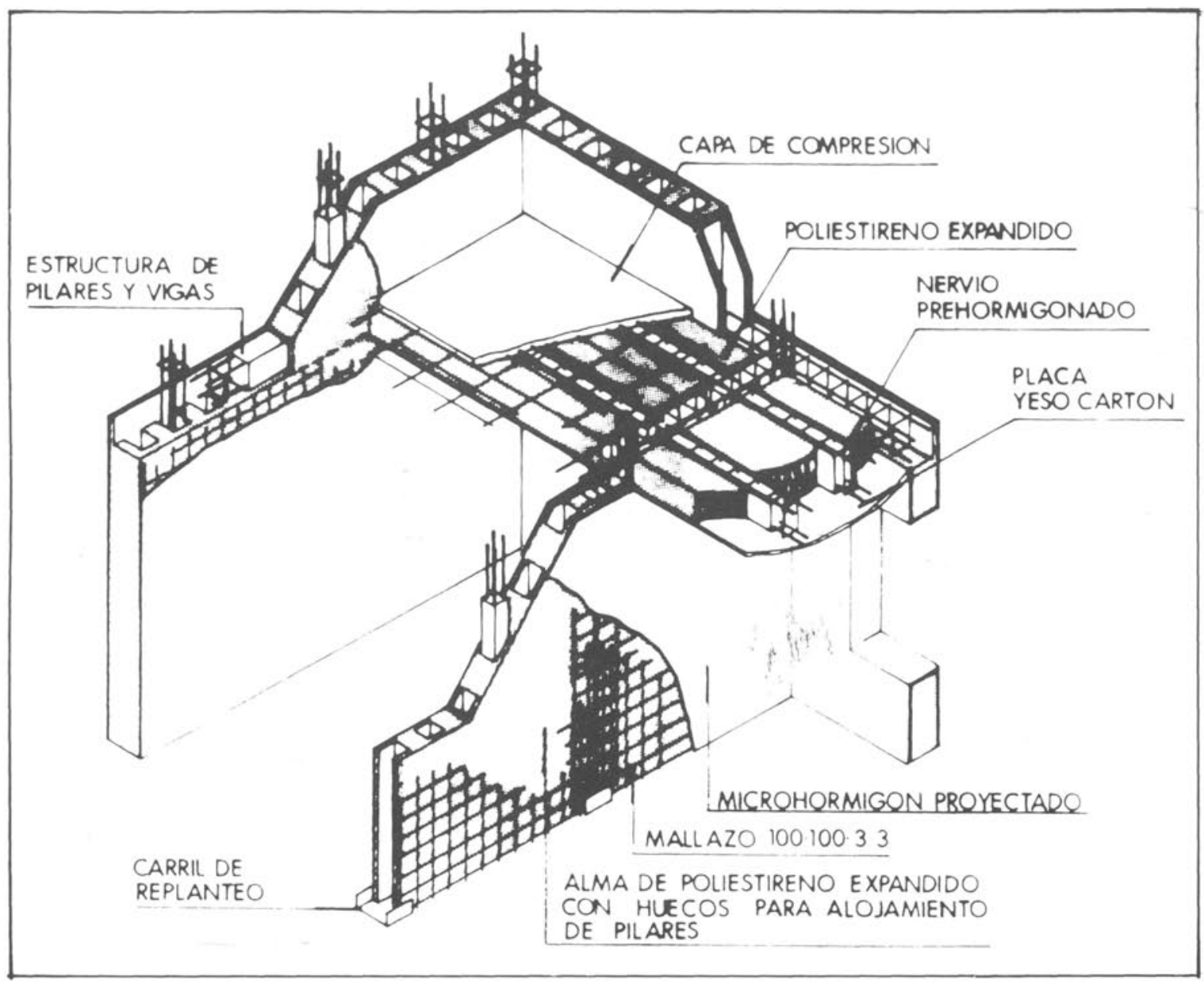

Fig. 11-Esquema de la estructura del sistema Dragados-Plastbau.

tuados en zonas de sismicidad importante. En los otros casos, los pórticos de hormigón deberán rigidizar el edificio ante las acciones horizontales, en ambas direcciones. Sin embargo, estudios recientes han puesto de manifiesto la posibilidad de poder contar con la colaboración resistente de las capas de microhormigón, tanto ante las acciones verticales como ante las acciones horizontales debidas al viento o al sismo.

El forjado se estudia como un elemento continuo que transmite las cargas a los pilares con una determinada excentricidad. Es preciso comprobar la tensión tangencial en los nervios, entre los hormigones de diferentes fases, y disponer armaduras de cosido cuando asi lo requiera el cálculo.

\section{ESTRATEGIAS PARA MEJORAR LA DURABILIDAD DE LAS ESTRUCTURAS}

\subsection{Consideraciones previas}

Las estructuras deben proyectarse, construirse y utilizarse de forma que se mantengan unos niveles aceptables de seguridad y funcionalidad, durante el período de vida previsto para las mismas, sin costes inesperados de mantenimiento y reparación.

Ese periodo de vida previsto o vida útil puede ser fijado explictamente por el propietario del edificio o puede estar contenido implicitamente en los códigos y pliegos de condiciones. Habitualmente, los códigos (2) (4) contemplan un periodo de 50 años para la vida útil de las estructuras de los edificios.

Algunas estructuras construidas defectuosamente o situadas en ambientes altamente agresivos se deterioran prematuramente y su seguridad o funcionalidad quedan comprometidas antes de los 50 años, lo que exige su reparación. En este sentido, tienen gran inte. rés práctico los trabajos que actualmente se llevan a cabo para desarrollar modelos que reproducen la evolución de la respuesta de la estructura deteriorada a lo largo del tiempo (18), con el fin de optimizar el mo. mento de su reparación.

En cualquier caso, conviene prevenir el daño en la es. tructura antes que éste haya generado un deterioro di. fícil y costoso de solventar. Interesa pues realizar inspecciones periódicas de las estructuras y utilizar téc. nicas de auscultación que permitan detectar rápida- 


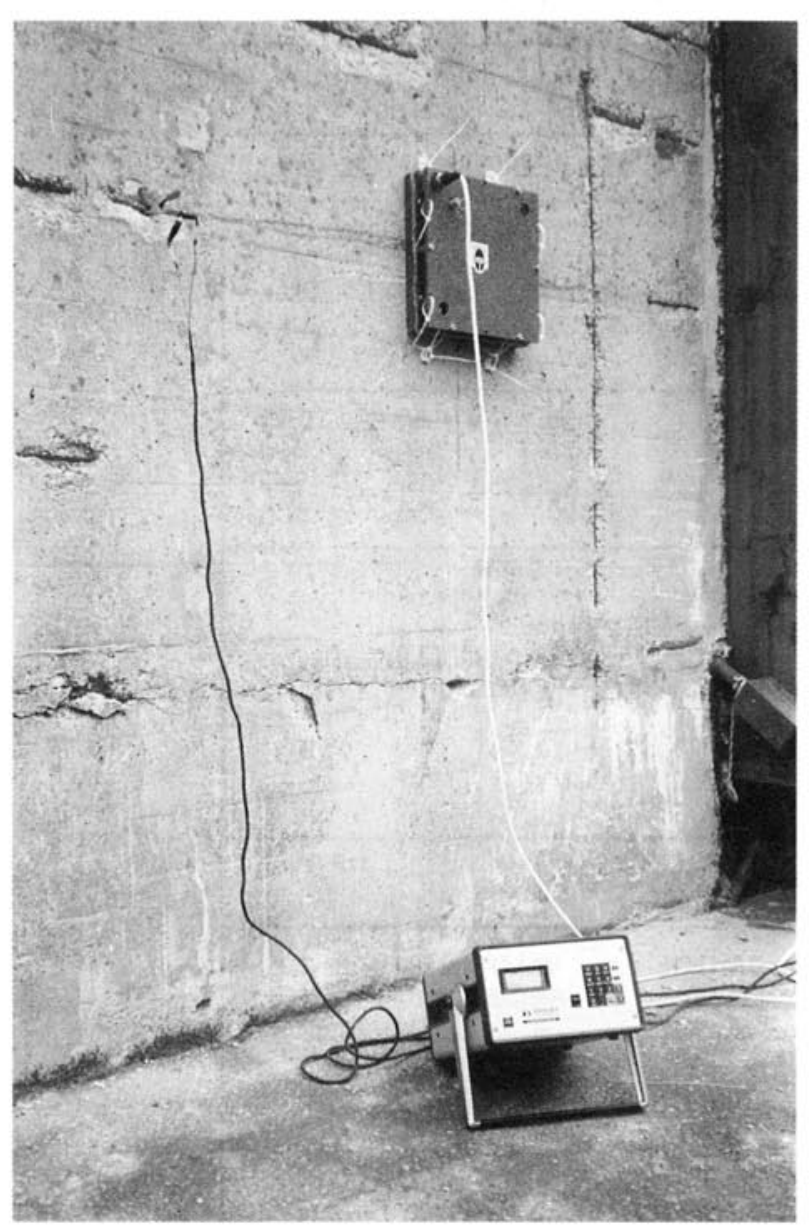

Fig. 12.-Corrosimetro para la medición de la velocidad de corrosión de las armaduras en estructuras de hormigón.

mente el deterioro iniciado. A título de ejemplo, el ponente de esta conferencia coordina un proyecto de investigación EUREKA/EUROCARE, que lidera la empresa GEOCISA y en el que también participan por parte española dos centros del CSIC (CENIM y TORROJA), en el que se están desarrollando corrosimetros portátiles para medir la velocidad de corrosión de las armaduras en estructuras de hormigón (fig. 12), aplicando técnicas de medida electroquímicas no destructivas. Estas mediciones permiten cuantificar la corrosión de las armaduras y adoptar soluciones antes de que el hormigón se haya fisurado o la sección de la armadura se haya reducido significativamente.

\subsection{Tipos de deterioro}

Las estructuras de hormigón se deterioran por diversas causas, destacándose aquellas que producen la corrosión de sus armaduras, sea ésta debida a la carbo. natación de la capa superficial del hormigón o a la acción de los cloruros en obras situadas en ambiente marino o en aquéllas en las que se emplean sales para el deshielo. Las estructuras también se deterioran por la acción del hielo o por acciones de tipo químico so- bre el hormigón, que al producir expansiones afectan a la integridad del mismo (ataque por sulfatos, reacción de cierto tipo de áridos con los álcalis del cemento,...).

Es esencial determinar el tipo de agresividad a la que estará sometida la estructura durante las distintas fases por las que pasa el edificio. Los códigos (2) (4) definen distintos tipos de ambientes en función del grado y tipo de agresividad. No obstante, estas definiciones distan mucho de ser satisfactorias, al ser fundamentalmente cualitativas, por lo que en el futuro será necesario definir los distintos ambientes de forma más precisa y cuantitativa.

\subsection{Medidas para mejorar la durabilidad}

En las fases del proyecto, construcción y mantenimiento de las estructuras de hormigón deben adoptarse medidas con el fin de mejorar su durabilidad. Seguidamente, se concretan estas medidas para el caso de la corrosión de las armaduras, aunque algunas son comunes a otros tipos de deterioros.

En la fase de proyecto, una vez conocida la vida prevista para la estructura y el ambiente en el que va a estar emplazada, deben adoptarse las medidas que a continuación se relacionan (19):

- Utilizar formas exteriores de la estructura que eviten la acumulación de agua en su superficie, facilitando su evacuación.

- Diseñar hormigones con contenidos en cemento no inferiores a $300 \mathrm{~kg} / \mathrm{m}^{3}$ y relaciones agua/cemento entre 0,45 y 0,60 , dependiendo del nivel de agresividad.

- Cuidar el detalle del armado de cada elemento, proyectando espesores del recubrimiento de hormigón acordes también con la agresividad ambiental (fig. 13).

- En los casos de fuerte agresividad, adoptar medidas de protección adicional en la armadura (galvanizadas o con resina epoxi), en el hormigón (empleo de inhibidores de la corrosión: nitritos,...), en la superficie de las estructuras (pinturas, recubrimientos,...) o aplicar técnicas de protección catódica (fig. 2).

Durante la construcción de la estructura deben también adoptarse algunas medidas:

- Control minucioso de la ejecución, que garantice las medidas adoptadas en el proyecto: colocación de la ferralla, calidad del hormigón,...

- Realización esmerada de la compactación y el curado del hormigón (fig. 1).

http://informesdelaconstruccion.revistas.csic.es 


\begin{tabular}{|c|c|c|c|c|c|c|}
\hline \multicolumn{7}{|c|}{ NORMA BRITANICA BS-8110 } \\
\hline \multicolumn{2}{|c|}{ TIPO DE AMBIENTES } & \multicolumn{5}{|c|}{ VALOR NOMINAL DEL RECUBRIMIENTO $(\mathrm{mm})$} \\
\hline \multicolumn{2}{|c|}{$\begin{array}{l}\text { SUAVE } \\
\text { MODERADO } \\
\text { AGRESIVO } \\
\text { MUY AGRESIVO } \\
\text { EXTREMO }\end{array}$} & $\begin{array}{l}25 \\
- \\
- \\
-\end{array}$ & $\begin{array}{l}20 \\
35 \\
- \\
-\end{array}$ & $\begin{array}{l}20\left(^{\star}\right) \\
30 \\
40 \\
50\left(^{* *}\right) \\
-\end{array}$ & $\begin{array}{l}20\left(^{*}\right) \\
25 \\
30 \\
40\left(^{* *}\right) \\
60\end{array}$ & $\begin{array}{l}20(*) \\
20 \\
25 \\
30 \\
50\end{array}$ \\
\hline \multicolumn{2}{|c|}{$\begin{array}{l}\text { MAXIMA AGUA LIBRE/CEMENTO } \\
\text { MINIMO CEMENTO EN } \mathrm{kg} / \mathrm{m}^{3} \\
\text { MINIMA RESISTENCIA DEL } \\
\text { HORMIGON EN } \mathrm{kp} / \mathrm{cm}^{2}\left({ }^{*}\right)\end{array}$} & $\begin{array}{r}0.65 \\
275 \\
300\end{array}$ & $\begin{array}{l}0.60 \\
300 \\
350\end{array}$ & $\begin{array}{r}0.55 \\
325\end{array}$ & $\begin{array}{r}0.50 \\
350\end{array}$ & $\begin{array}{r}0.45 \\
400 \\
500\end{array}$ \\
\hline \multicolumn{7}{|c|}{ 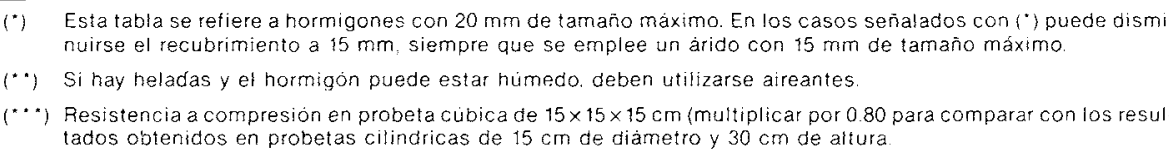 } \\
\hline \multicolumn{7}{|c|}{ NORMA JAPONESA JASS 5} \\
\hline AMBIENTE & \multicolumn{2}{|l|}{ TIPO DE ELEMENTO } & $\begin{array}{l}\text { VIDA UTIL } \\
\text { (años) }\end{array}$ & \multicolumn{3}{|c|}{$\begin{array}{l}\text { VALOR NOMINAL DEL } \\
\text { RECUBRIMIENTO }(\mathrm{mm})\end{array}$} \\
\hline \multirow[t]{2}{*}{ INTERIOR } & \multicolumn{2}{|l|}{ muros y losas } & $\begin{array}{r}65 \\
100\end{array}$ & \multicolumn{3}{|c|}{$\begin{array}{l}30 \\
40\end{array}$} \\
\hline & \multicolumn{2}{|l|}{ pilares y vigas } & $\begin{array}{r}65 \\
100\end{array}$ & \multicolumn{3}{|c|}{$\begin{array}{l}40 \\
50\end{array}$} \\
\hline \multirow[t]{2}{*}{ EXTERIOR } & muros y losas & & $\begin{array}{r}65 \\
100\end{array}$ & \multicolumn{3}{|c|}{$\begin{array}{l}40 \\
50\end{array}$} \\
\hline & pilares y vigas & & $\begin{array}{r}65 \\
100\end{array}$ & \multicolumn{3}{|c|}{$\begin{array}{l}50 \\
60\end{array}$} \\
\hline
\end{tabular}

Fig. 13. - Valores del espesor del recubrimiento de hormigón en función de los tipos de ambientes

Finalmente, es necesario realizar un mantenimiento de la estructura con un programa de inspecciones periódicas que debe planificarse en la etapa del proyecto, estudiando el estado de la estructura y sus acabados, si éstos están previstos como protección adicional de la misma.

\section{REFERENCIAS BIBLIOGRAFICAS}

1. J. Rodriguez: "El empleo de las cenizas volantes en la fabricación de los hormigones". Revista de Obras Públicas, pags. 663 a 676 . Julio 1988.

2. Comisión Permanente del Hormigón: "Instrucción para el proyecto y la ejecución de obras de hormigón en masa o armado. EH-88". MOPU. 1989.

3. AENOR: "Barras corrugadas, de acero soldable, para armaduras de hormigón armado". Norma UNE 36068. Asociación Española de Normalización, 1988.
4. CEC: "Eurocode No.2. Design of Concrete Structures. Part 1: General Rules and Rules for Buildings". Commission of European Communities. Revised Fi. nal Draft. Dec. 1989.

5. C. Andrade: "Manual para inspección de obras danadas por corrosión de armaduras". Instituto de Ciencias de la Construcción E. Torroja. Consejo Superior de Investigaciones Cientificas. Madrid, 1989.

6. J. Rodríguez: "Morteros de cemento reforzados con fibra de vidrio". Revista BIA. Colegio de Aparejadores y Arquitectos Técnicos de Madrid. Julio, 1987.

7. S. P. Shah, G. B. Baston: "Fiber Reinforced Concrete. Properties and Applications". SP-105. American Concrete Institute.

8. R. Walther: "Potenciality of Utilizing High Strength Concrete in Structures'. Bulletin d'Information n. 193, pp. 101-117. CEB Comite Euro-International du Beton. 1989.

http://informesdelaconstruccion.revistas.csic.es 
9. FIP/CEB: "High Strength Concrete. State of the Art Report". SR 90/1, FIP or Bulletin d'Information n. 197, CEB. 1990.

10. C.S.T.B.: "Parois et murs en béton banché. Regles de calcul". D.T.U. n. 23.1. Paris, 1975.

11. C.S.T.B.: "Dalles pleines confectionnéss à partir de predalles préfabriquées et de béton coulé en oeuvre. CPT Planchers". Paris, 1979.

12. B. O. Aalami: "Design of Post-Tensioned Floor Slabs". Concrete International, Jun. 1989, pp. 59-67. American Concrete Institute.

13. $\mathrm{ACl}$ 423: "Recommendations for Concrete Members Prestressed with Unbonded Tendons". ACI Structural Journal, May 1989, pp. 301-318. American Concrete Institute.

14. FIP: "Recommendations for the design of flat slabs in post-tensioned concrete (using unbonded and bonded tendons)". FIP /2/5. May 1980.
15. P. Díaz, A. Ruiz, J. Flórez: "Sistema DragadosPlastbau". Informes de la Construcción n. 383, pp. 5-9. I. E. Torroja. 1986.

16. J. Quereda, E. Rúa, J. M. Conde, J. Rodríguez: "Estabilidad estructural del sistema DragadosPlastbau". Informes de la Construcción n. 383 , pp. 25-35. I. E. Torroja. 1986.

17. C. Andrade, J. Rodríguez: "Durabilidad de las armaduras en el sistema constructivo DragadosPlastbau". Informes de la Construcción n. 383, pp. 49-53. I. E. Torroja, 1986.

18. J. Rodriguez, C. Andrade: "Load-bearing capacity loss in corroding structures". ACl Spring Convention. Toronto. Canada. March 1990.

19. CEB: "Durable Concrete Structures CEB Design Guide". Bulletin n. 182. Comite Euro-International du Beton. Jun. 1989.

\title{
publicación del ICCET/CSIC
}

\author{
INSPECCION DE OBRAS DAÑADAS \\ POR CORROSION DE ARMADURAS
}

El presente Manual va dirigido principalmente a técnicos especializados y laboratorios que tienen que intervenir en el dictamen de la situación de deterioro de estructuras de hormigón armado dañadas por corrosión de armaduras.

Comienza con un resumen recordatorio de los factores principales a los que se pueden deber los daños prematuros por corrosión de armaduras, para seguir con algunas indicaciones de cómo se deben realizar las inspecciones, y de los ensayos y la metodología que se recomienda realizar para poder dictaminar con precisión las causas de daño.

A continuación se hacen una serie de comentarios sobre la vida residual de estructuras dañadas, sobre el riesgo de corrosión futura, el seguimiento necesario de una estructura reparada y una breve enumeración de métodos de reparación y consideraciones básicas a tener en cuenta en la recomendación de un determinado método. Se aporta una breve relación bibliográfica.

Finalmente se incluyen en forma de ficha la descripción de algunos casos de corrosión de armaduras detectados en nuestro país.

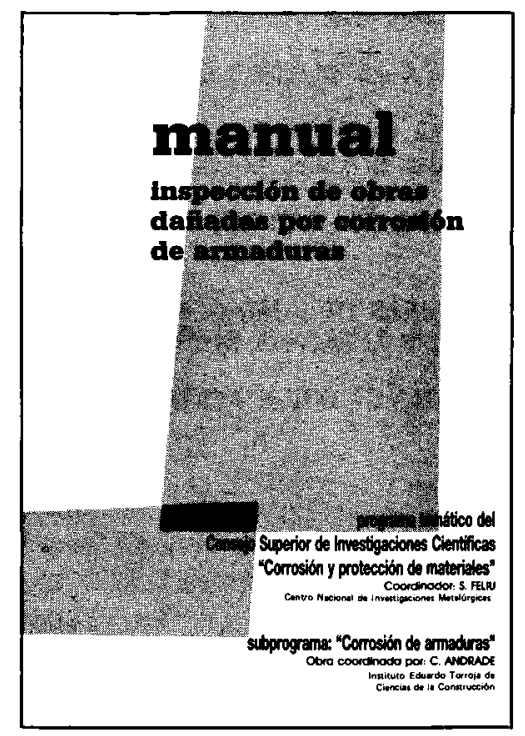

